\title{
PRECISE RADIONUCLIDE LOCALIZATION USING UAV-BASED LIDAR AND GAMMA PROBE WITH REAL-TIME PROCESSING
}

\author{
S. Schraml ${ }^{1 *}$, T. Hinterhofer ${ }^{2}$, M. Pfennigbauer ${ }^{2}$, M. Hofstätter ${ }^{1}$ \\ ${ }^{1}$ AIT Austrian Institute of Technology GmbH, Giefinggasse 4, 1210 Vienna, Austria - (stephan.schraml, \\ michael.hofstaetter)@ait.ac.at \\ ${ }^{2}$ RIEGL Laser Measurement Systems GmbH, Riedenburgstr. 48, 3580 Horn, Austria, - (thinterhofer, mpfennigbauer)@riegl.com
}

KEY WORDS: UAV, lidar, 3D point cloud, radiation, real-time, first responders, source localization

\begin{abstract}
:
In this work we propose an effective radiation source localization device employing a RIEGL VUX-1UAV laser scanner and a highly sensitive Hotzone Technologies gamma radiation probe mounted on a RiCOPTER UAV combined with real-time data processing. The on-board processing and radio communication system integrated within the UAV enables instant and continuously updated access to georeferenced 3D lidar point clouds and gamma radiation intensities. Further processing is done fully automated on the ground. We present a novel combination of both the 3D laser data and the gamma readings within an optimization algorithm that can locate the radioactive source in real-time. Furthermore, this technique can be used to estimate an on-ground radiation intensity, which also considers the actual topography as well as radiation barriers like vegetation or buildings. Results from field tests with real radioactive sources show that single sources can be located precisely, even if the source was largely covered. Outcomes are displayed to the person in charge in an intuitive and user-friendly way, e.g. on a tablet. The whole system is designed to operate in real-time and while the UAV is in the air, resulting in a highly flexible and possibly life-saving asset for firstresponders in time-critical scenarios.
\end{abstract}

\section{INTRODUCTION}

When order is suddenly suspended, there's not much left to rely on, but the courage and experience of rescue task forces. Their routine, in fact, is to face the unknown, every time they enter a terrain, where a disaster has struck. Steady efforts to implement latest technological means to support their work aim at securing task forces' working conditions, such as accelerating search for orphan radioactive material and make the whole rescue mission more efficient. Besides of promising methods to guide an observer (Ristic et al., 2010), great effort has been put into localizing radioactive sources by mobile systems (Ardiny et al., 2019). While (Gordon et al. 2017) relies on a UAV equipped with an imaging system and a radiation probe to navigate a UGV, we propose a system that is solely based on using a UAV carrying a gamma sensor. Much in this context is expected from using autonomous vehicles for obvious reasons: to go where it would be too dangerous or even impossible for manned aircraft to go, has always been one of the major arguments for UAV utilization. Especially equipped UAVs have already been proposed for fighting fires, for carrying out search missions, for delivery of rescue kits, and many other impressive tasks. UAV reconnaissance is considered a very powerful way of overcoming the dangerous lack of information in danger areas.

This work reports about an experiment carried out within the framework of the Austrian research project SecuRescue. The following emergency scenario was imagined for a test set-up: a disaster with the potential for nuclear contamination, caused by natural forces or technical failure, hits an urbanized and partly industrial area. Immediate effect: a complete destruction of the topographic array caused by collapsed buildings. Available existing maps are useless. The test focused on how to provide essential information to first responders as quickly as possible, or ideally, in real time. Furthermore, the location of the radioactive source was to be identified.

As depicted in Figure 1, all information, i.e. the up-to-date 3D terrain and the radioactive nuclide localization should be presented to first responders on a handheld tablet in an intuitive and user-friendly way.

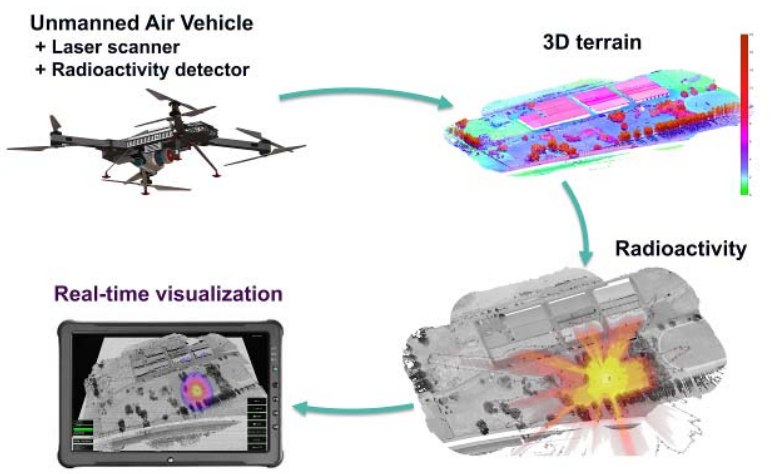

Figure 1: Approach for UAV-based 3D terrain acquisition and radionuclide localization.

\section{HARDWARE EMPLOYED}

\subsection{UAV}

The complete sensor payload consisting of laser a laser scanner and radiation sensor was integrated on RiCOPTER-M (c.f. Figure 2), a 40-kg electrical octocopter with $15 \mathrm{~kg}$ maximum sensor payload, thus perfectly prepared for the integration of multi-sensor systems in interchangeable configurations. The

* Corresponding author 
high performance UAV (flight time up to 30 min) has been especially designed for operation in particularly sensitive areas. It can optionally be equipped with LED projectors for search missions at night, an infrared camera, or other sensors. For the present test setup, flights were carried out in daylight and under fair meteorological conditions.

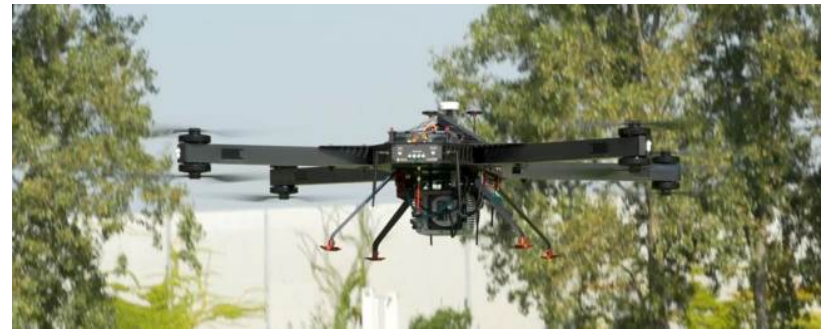

Figure 2: RIEGL RiCOPTER-M during test flight

As the RiCOPTER-M is to be safely operable even above populated areas, it is resilient to the main single failures. To achieve this, two completely independent flight controllers with separated power supply and dedicated sensory hardware (i.e. GNSS, gyroscope, accelerometer, and compass) are integrated. The sophisticated design permits utmost reliable operation, yet, while expectations for UAV use in disaster management are high, there's still concern that they could present a disturbance, if not a threat to rescue teams, especially with regards to coordination with manned search and rescue helicopters.

For this reason, the RiCOPTER-M, registered call sign „OEVUX“, can be operated with an ADS-B/Mode S transponder (http://www.sagetech.com), in coordination with air traffic management. Sure, the rules of the air clearly dictate all-time priority for manned aircraft, and the UAV's flightpath is to be chosen accordingly. But while it's good to have top priority, it might still be reassuring for pilots to have a UAV in their vicinity clearly displayed on their screen, and the possibility to rely on ATM warnings or separation.

\subsection{Laser scanner}

The RIEGL VUX-1UAV (c.f. Figure 3) laser scanner was used for acquiring the precisely updated topographic information.

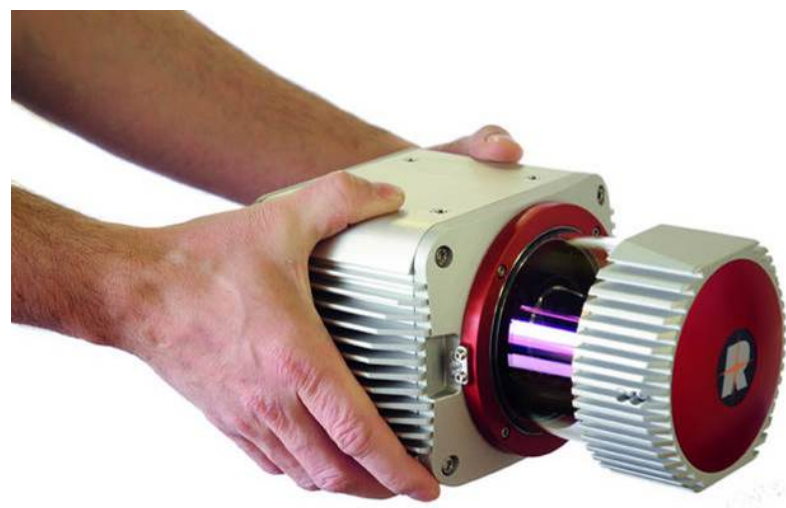

Figure 3: RIEGL VUX-1UAV laser scanner

The VUX-1UAV is a survey-grade laser scanner with a rotating mirror, a field-of-view of $330^{\circ}$ and a measurement rate of up to 500.000 measurements per second. It is a lightweight and compact laser scanner, meeting the challenges of UAV survey solutions, both in measurement performance as in system integration. Usually, the measurement data of the VUX-1UAV is stored on an internal SSD drive and processed offline after data acquisition. With the according post-processing toolchain, a measurement accuracy of $10 \mathrm{~mm}$ is achieved.

\subsection{Gamma spectrum probe}

The gamma spectrum probe, GIHMM GSP02, provides gamma radiation data in "ambient dose equivalent rate" and supports spectroscopic identification of gamma radiation which facilitates on-site identification of isotopes. It has a large measurement range and low detection thresholds. Its weight was significantly reduced by using a customized lightweight housing with a volume of $420 \mathrm{ml}$. The radiation measurement is based on a plastic-scintillator with attached photomultiplier. Gamma and beta radiation hitting the crystal is displayed as pulses, which are detected and converted to Counts Per Seconds (CPS) by an internal processor. The current CPS are continuously provided every $250 \mathrm{~ms}$ over a serial communication port. This measurement rate enables a highresolution acquisition of the local radiation pattern.

\subsection{Real-Time Georeferencing}

2.4.1 Laser scanner: Conventional post-processing of the LiDAR data allows to access and to analyze the entire information acquired. In situations as the presently described one, however, it is time that counts, and the availability of immediate information to safely access the terrain. Reliable and precise data acquisition being the well-proven capacity of stateof-the-art LiDAR survey, the real-time transmission of only that part of information relevant for orientation and localization purposes out of the enormous amount of data was the tricky part. Therefore, the real-time data processing approach relied on a completely different method. As depicted in Figure 4, an embedded processing system integrated in RiCOPTER-M immediately processed the raw laserscanning data (i.e. distance measurements in the SOCS Scanner Own Coordinate System) with the real-time trajectory (i.e. position and orientation) generated by the GNSS/IMU system. The result was the generation of a real-time 3D point cloud in a global reference coordinate system (WGS 84). The GNSS system had no access to any kind of correction signal resulting in an absolute height error of up to $2 \mathrm{~m}$.

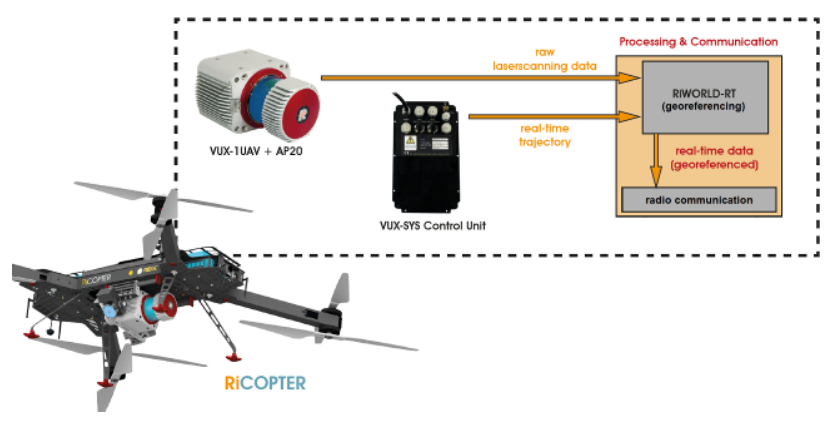

Figure 4: Embedded processing system integrated in RiCOPTER-M

In addition, the georeferenced laserscanning data was converted to a binary data format, which is tolerant to wireless communication outages enabling the transmission over the widely used UDP/IP protocol. As a result, the data stream can be transmitted even over unreliable wireless connections without affecting data acquisition. Transient communication issues (channel busy, fast fading, etc.) do not cause 
disconnections or high transmission delays but only limited data loss on ground. As soon as the connection is re-established, real-time data will be available again. With the described approach, a net rate of 50.000 acquired and real-time-processed measurements per second was achieved. This results in a bandwidth requirement of roughly $10 \mathrm{Mbit} / \mathrm{s}$ for the radio communication link to the ground-station.

2.4.2 Gamma sensor: As the gamma probe outputs measurements only, which do not contain any information about their location and time, a GNSS / IMU system integrated on the UAV was used to deliver navigational data during flight, which enables the localization of the measured data as described in 3.3. This system sends the accurate trajectory (position and location) over the TCP / IP protocol.

\section{RADIATION SOURCE LOCALIZATION}

The use of unmanned vehicles for supporting the important task of mapping gamma radiation levels and the localization of a radiation source is advantageous for many reasons, most of all for health issues. UAVs showed to be beneficial (Gabrlik, Lazna, 2018) specially when the terrain is difficult to access. However, we aim to go further and precisely localize a source on ground through modelling of measured values, which gives an additional opportunity to estimate radiation levels on ground, for e.g. finding safe passages.

By aiming to localize a radioactive source in a terrain, following assumption was made: The source is at least partially visible, which means that it is neither buried deep in soil nor hidden inside a building or a container. This is essentially equivalent to the presumption that the source is located on ground. However, we consider that vegetation may be present, which has a shielding effect on radiation due to its water content. To correctly model that effect, one first important step in our model is to differ between soil and vegetation. An overview of the methodology to achieve a precise radionuclide localization in real-time is presented in Figure 5:

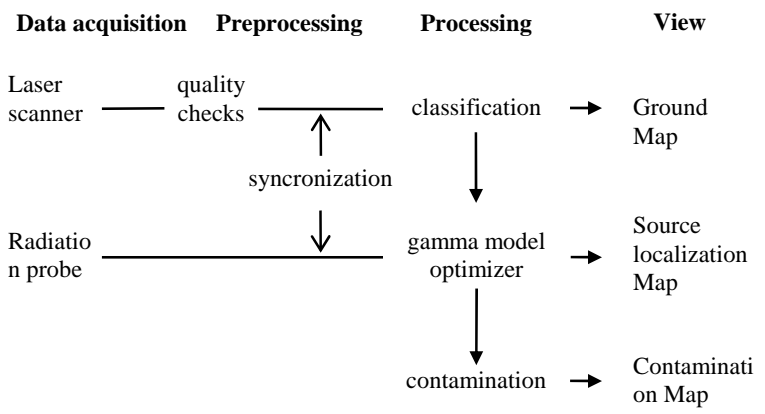

Figure 5. Scheme of process followed to localize a radiation source on ground.

In order to efficiently support first-responders when arriving in a disaster area, we aim to reveal the real location of a single gamma source instead of showing the measured raw data solely. At first received data is cleared from outliers and gamma values are georeferenced. Then a classification module determines which of the points belong to vegetation, such that a solid ground model can be established. Based on this a gamma model is optimized to determine the source location. The processing provides following results: i) the ground model, ii) the location of the gamma source and iii) a contamination map. Furthermore, those results are attempted to be provided right away by following two principles: i) Data is processed immediately as it is acquired. ii) Laser scanner and gamma sensor data is combined with existing measurements, refining the results with more details and accuracy as the UAV continues to scan over the targeted area.

\subsection{Data acquisition}

Laser scanner data is received as a continuous stream of georeferenced 3D points, where each point is supplemented with attributes such as amplitude, reflectivity and timestamp. In general, point density on ground can vary as it depends on UAV's flight parameters like altitude and speed. Moreover, typical flight pattern includes meander and eight-shaped loop patterns resulting in scan-overlaps and thus in an increased point density of those areas.

The radiation probe data consist of only the periodic CPS measurements. Localization of these measurements is done on ground by using the transmitted UAV position.

\subsection{Quality checks}

The laser scanner occasionally observes points in the atmosphere that do not stem from rigid objects and can appear randomly. Those have a potential to inflict the outcome of gamma model processing and should therefore be removed. A prior task is to filter all those points that are very likely not to belong to a physical object on ground. We use a set of criteria based on properties of individual points, namely amplitude, reflectivity, distance to sensor and height. More complex filter techniques based on correlation between sets of points were dismissed due to performance reasons and the good efficiency of the simple point-based filter.

\subsection{Synchronization}

The gamma model to be processed is based on the spatial pattern of radiation values. Since the radiation probe delivers radiation measurements only and does not provide timestamp or position information by itself, a precise localization must be achieved afterwards. For that reason, the UAV send additionally to the laser measurements also navigational data at short intervals and gamma values are timestamped on ground, which are then combined by using correlation of respective timestamps. Though, the processing is not done on board (of the UAV), the achieved georeferencing is of sufficient accuracy, keeping in mind that gamma readings are at relatively long intervals compared to transmission and synchronization time.

\subsection{Classification}

The use of airborne laser scanners to determine a ground elevation model has attracted intensive research (Meng et al., 2010) for many reasons. Among them are the high accuracy and resolution of Lidar point clouds that enable accurate ground models. In order to build a ground model based on Lidar a first step is to identify ground points that are located lowest within a small area, whereas higher laser points may result from either vegetation or reflections from e.g. dust in the air. Subsequently, by removing the vegetation points the underlying ground structure will be revealed.

Therefore, we apply an iterative classification algorithm to mark points as vegetation based on two criteria: i) Points that do have close neighbours (in terms of $x-y$ coordinates) with a lower height value (z-coordinate) are classified as vegetation. Note, this implies to determine the context of a point appearance and thus to investigate the local neighborhood of 
each 3D point. ii) By making use of the specific laser scanner capability to detect multiple targets per laser shot, only the last targets in terms of distance to the scanner may be considered as ground points. It has to be noted, that thereby manmade buildings will be recognized as solid ground too.

One of the challenges when searching for ground points, is fixing the size of the relevant local area for the first criteria. It can be observed that in areas with vegetation, typically 3D points are created from both vegetation and ground. However, in areas of very dense vegetation, only a few, if any, laser shots reach the ground. Those points are needed to guide the algorithm when building the ground model. While a small search area will not allow to find ground points in a dense vegetation, a large search area will cause a loss in detail. Furthermore, due to the data acquisition process point density variations will appear naturally, where highest density is directly below the scanner. In our classification, the size of the search window is therefore adaptively guided by local properties, e.g. point density and vertical spread to account for density variations. That way, the search window is kept small on solid ground while in areas of vegetation it is large to increase the probability of detecting a ground point. Then, a ground model can be found by simply removing all points whose classification was set to vegetation.

An example is shown in Figure 6 with results of the vegetation filter on 3D data of a cross section of a typical environment including plain areas, vegetation and buildings. As the adaptive window becomes smaller on areas with high point density, small bumps can successfully be recovered (1), while on the other hand a large adaptive window allows to cover large data gaps (4). Buildings are consequently identified as solid ground (3). Note, the small tree between these two buildings is correctly identified as vegetation (2).

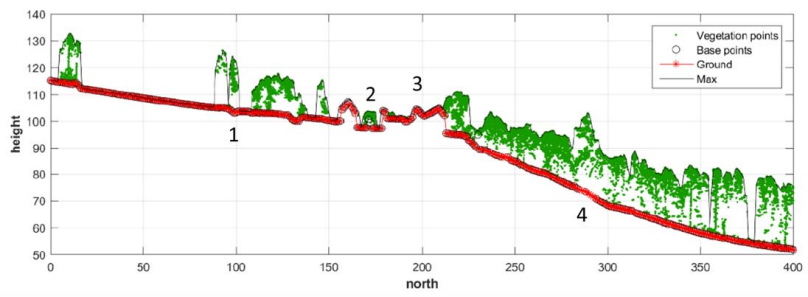

Figure 6: Vegetation filter on a typical terrain, showing the results for a cross section.

\subsection{Gamma model optimizer}

This module aims at finding the localization of a hazardous source. As earlier mentioned, the source is assumed to be on the surface. Furthermore, it is assumed that at most one radiation source is present.

The general idea followed is firstly, to formulate a model that allows estimation of gamma values at locations of the actual gamma measurements under the presumption of the given terrain map and a specific source position. Secondly, it is stated, that such a model using the true gamma location as input would best explain the gamma measurements in terms of gamma value deviation. Thus, by iteratively testing different source locations it is possible to find a model, which proposes gamma values that minimally deviate from true measurements. This model is considered as indicating the real source location.

The radiation source parameters to be determined include the relative strength of the source $\left(\mathrm{I}_{0}\right)$, which is meaningful only as a relative measure and may not be used to determine the actual source strength, and the position on the terrain $(x, y)$, considering the height $\mathrm{z}$ is already given by the ground map. Calculations are based on the ground map, vegetation and gamma measurement data.

According to the inverse-square law, radiation intensities decline due to the spherical spread with the squared distance:

$$
\begin{aligned}
& I_{d}=\frac{I_{0}}{d^{2}} \\
& \text { where } \quad I_{0}=\text { radiation source intensity } \\
& I_{\mathrm{d}}=\text { radiation intensity at distance } \mathrm{d} \\
& \mathrm{d}=\text { distance }
\end{aligned}
$$

In addition, the traversed material, including air, cause attenuation of the gamma beams depending of the traversed material. We consider two materials: air and another one for soil and vegetation. Thus, the radiation intensity at a certain distance is calculated according to the Lambert-Beer law

$$
I_{z}=I_{d} * e^{-\mu^{*} z}
$$

where $\quad I_{d}=$ radiation intensity at distance $d$

$\mathrm{I}_{\mathrm{z}}=$ attenuated radiation intensity

$\mathrm{z}=$ traversed distance

$\mu=$ attenuation coefficient

Because a radiation beam may traverse different materials, we need to calculate the combined attenuation coefficient by splitting the exponent into parts according to the traversed materials:

$$
\mu^{*} z=\mu_{1}^{*} z_{1}+\mu_{2}^{*} z_{2}+\ldots+\mu_{n}^{*} z_{n}
$$

where $\quad \mathrm{z}=$ traversed distance

$\mu=$ attenuation coefficient

$\mathrm{l}_{\mathrm{i}}=$ length traversed materials

$\mu_{\mathrm{i}}=$ attenuation coefficients for materials

$\mathrm{n}=$ number of traversed parts

In order to find the source position following optimization is performed:

Repeat for all positions or until the cost function (eq. 4) becomes minimal

1. Choose the next position $(\mathrm{x}, \mathrm{y})$ on the ground

2. Calculate the minimal source strength by assuming a transparent medium, i.e. the attenuation coefficient $\mu=0$, such that all estimated gamma values are equal or greater than the measured ones.

3. Calculate a damping profile: based on the chosen position the attenuation factors $\mu$ for all gamma measurement positions are evaluated according to eq.2 and eq.3.

4. Estimate gamma values using that damping profile.

5. Calculate the error term.

The cost function consists of three weighted terms:

$$
f\left(I_{0} x, y\right)=\lambda_{1} * f_{\text {gamma }}+\lambda_{2} * f_{\text {damping }}+\lambda_{3} * f_{\text {strength }}
$$

The summed difference between estimated and measured gamma values build the first cost term. However, this does not incorporate the damping effect, which is observed when sources are partially obstructed or blocked, resulting in high differences that consequently increase the overall cost. We therefore add a second cost term measuring the differences between the 
expected damping profile based on the ground and vegetation information and the calculated damping profile, which is based on the measured gamma values. Since, we calculate the initial gamma values as always exceeding the measured ones, the difference between these two profiles can be explained as an additional attenuation effect. Finally, we add a term to penalize strong sources.

Using this procedure any optimization strategy, e.g. gradient descent, can be used to find the optimum model, indicating the source location.

\subsection{Contamination}

Based on the parameters, source location and source strength, found in previous steps, a fictive contamination map can be developed. Such a map contains estimations of radiation levels for each point of the terrain, for e.g. $2 \mathrm{~m}$ above ground.

A grid of fictive measuring points is laid out. Subsequently, for each point the expected radiation level is calculated using above formulas. Again, we consider the damping effect of radiation from two materials. It turns out, that basically it is possible to use the exact same methods from previous steps, the estimation of gamma values at any point. The only difference is that previously the estimation where made for locations of gamma measurements, whereas the locations are now in form of a grid over the target area. Since the damping effect strongly depends on the material as well as on the radiation source, the residual contamination map can only provide an estimation for those specific parameters.

\section{RESULTS AND DISCUSSION}

The tests were conducted on a region of about $350 \times 150 \mathrm{~m}$, showing mostly flat surface and scattered distinct areas with dense vegetation.

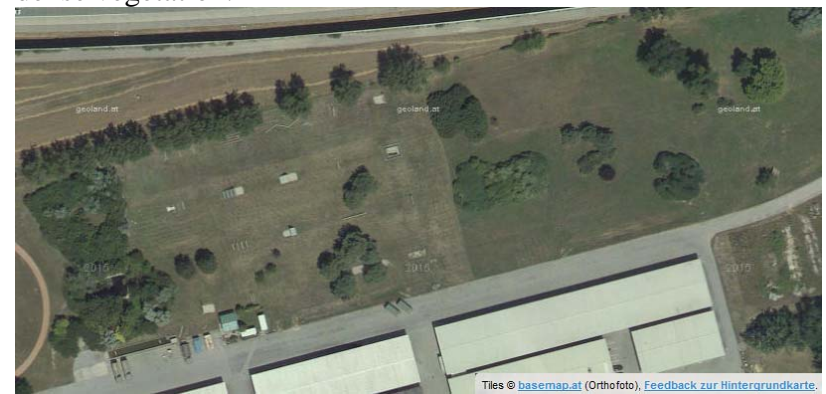

Figure 7: Image from test region

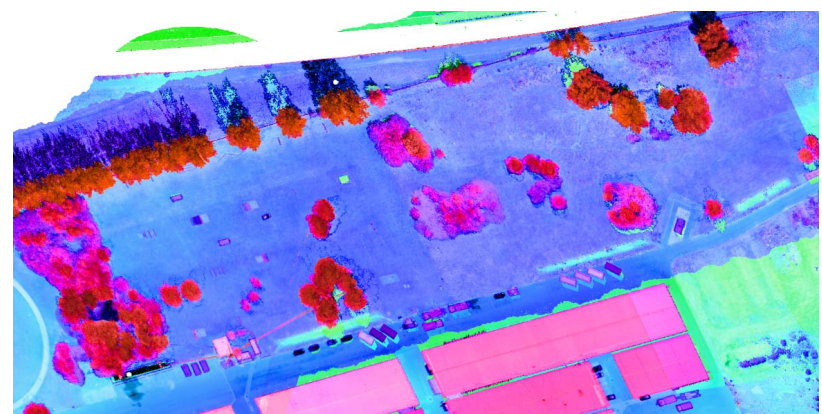

Figure 8: Laserscan data color-coded with height and reflectivity as color brightness.
4.1.1 Vegetation filter: Figure 9 shows the result of the vegetation filter for the same area. Trees and bushes are effectively removed, revealing the underlying terrain. For very dense and low growing vegetation it can occur that laser points do not reach the ground but vegetation points very close to the ground and therefore are sometimes misinterpreted, resulting in a rough ground model. E.g. are the bushes in the center and the left bottom corner. However, since these points are already close to the terrain it does not affect the source localization.

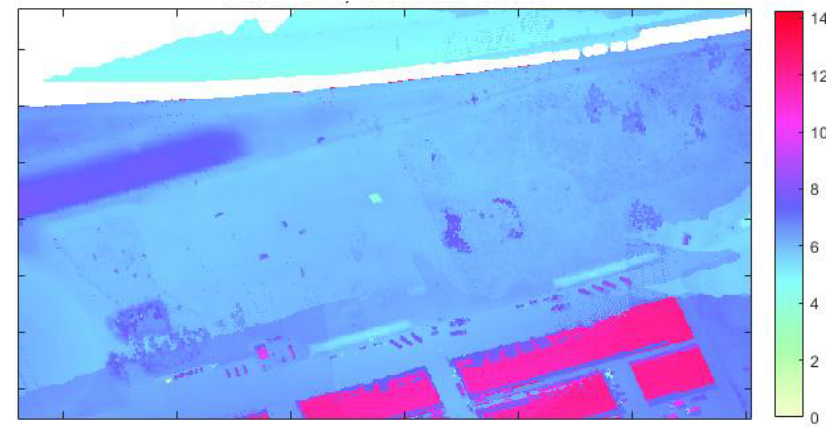

Figure 9: The same terrain with vegetation being removed. The height is color-coded.

4.1.2 Gamma model: Examples of measured and located gamma values are shown in Figure 10 in form of a color-coded trajectory of the UAV's path for two missions (A, B). In mission A, a source was placed on the street, plainly visible from above. The radiation beams were not blocked. Mission B was more difficult: A source was mainly covered with blocks of plumb, simulating an obstructed source. Only a few narrow beams could pass through, visible in the inhomogeneous distribution of gamma intensities. The color codes correspond to the measured gamma intensity (from blue to red with increasing intensity). For both missions the copter flew the same pattern in three different heights.

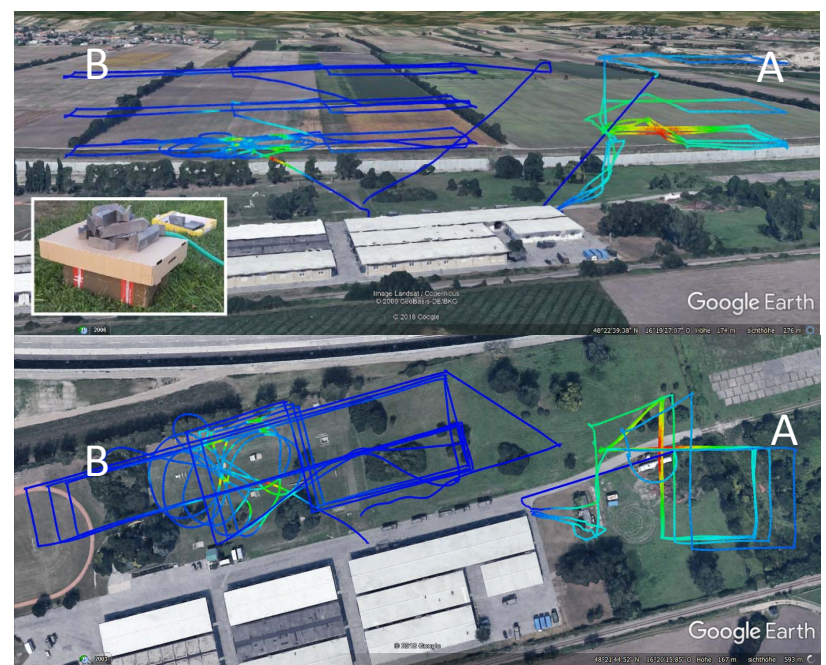

Figure 10: Gamma measurements for two different flights shown together viewed from side (top) and from birds-view (bottom). The measured gamma intensities are color-coded from low value (blue) to high values (red).

Mission A: The highest intensity measurements are in the middle of the lowest flight pattern. This simple example indicates that the gamma source position is almost directly below the crossing of the flight path. 
Mission B: The second example, on the left, shows the more complex situation, where the source is mostly covered. Only a few weak gamma beams were detected, making it hard to pinpoint the source by intuition since in there is no visible center as in the first example.

According to above described optimization procedure to find a model that can best explain the measurements, a source localization map was build. Basically, that map shows values of the cost term for each calculated position in a blueish color, see Figure 11. High cost values are transparent, while low cost values are blue to green. The color can be interpreted as likelihood of the source being at a certain position according to our model.
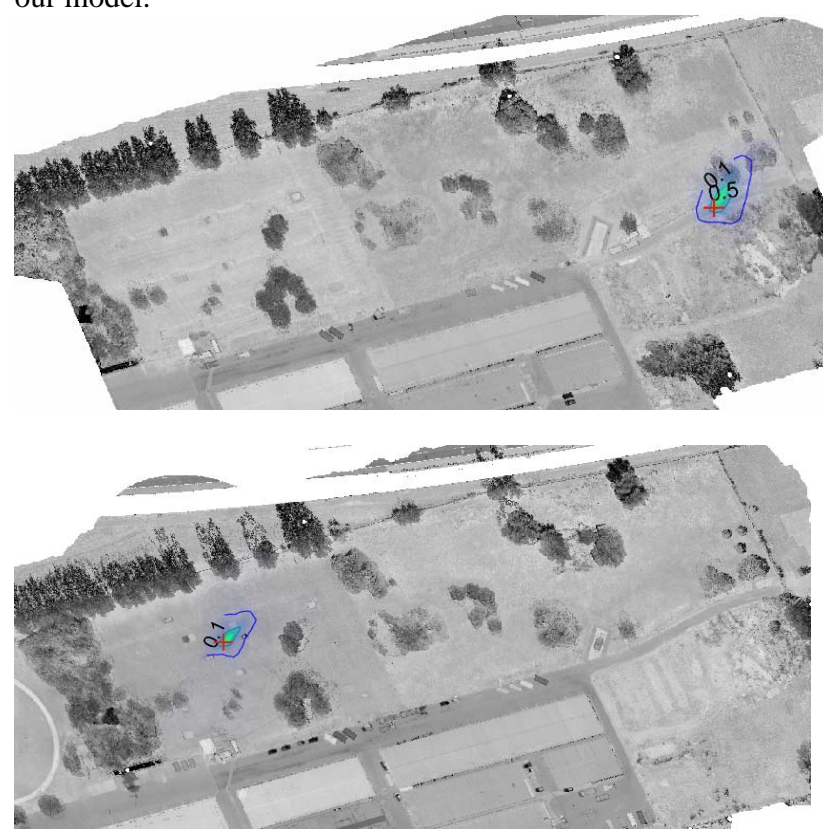

Figure 11. Result of source location processing, scenario A (top), scenario B (bottom).

In both examples the expected gamma location is within a few meters away from its actual location (red cross).

It also turned out that measuring gamma values from three different altitudes, though using the identical flight pattern again, greatly improved the accuracy.

4.1.3 Gamma contamination model: Using the point with the highest probability as source location we calculate the contamination map, i.e. estimate for each point above ground a radiation level. Since hills and vegetation will have a damping effect on the radiation we expect to estimate a lower level behind those hills and vegetation, as can be seen in Figure 12 and Figure 13.

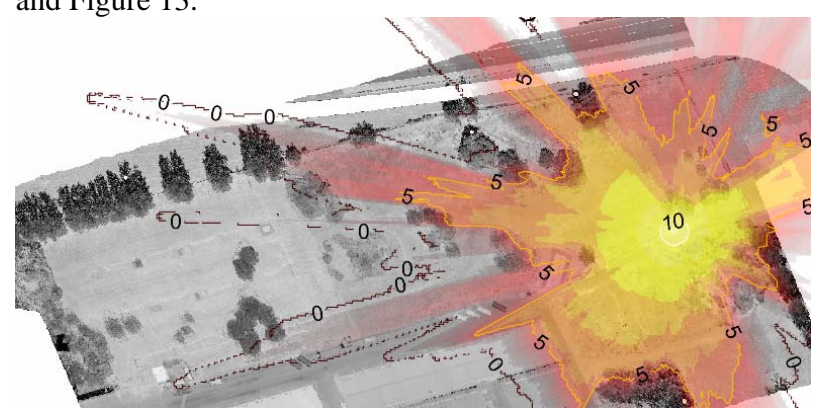

Figure 12. Estimated contamination map, radiation levels based on source location and constructed ground model are shown in log scale and color-coded, scenario A.

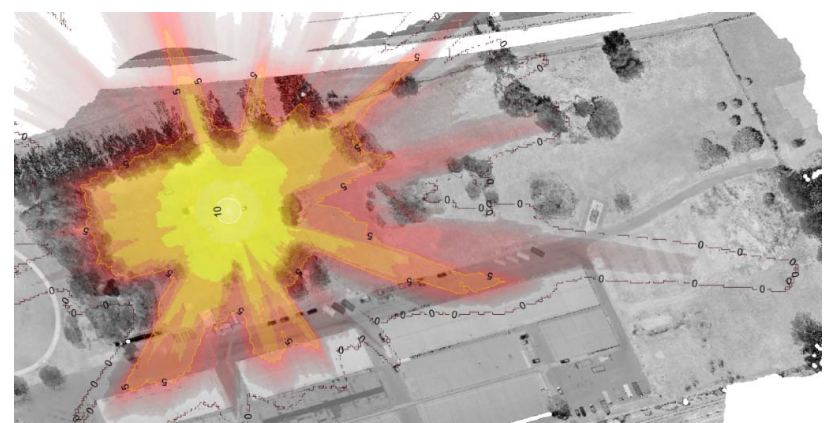

Figure 13. Estimated contamination map, scenario B.

\section{CONCLUSIONS}

The main conclusion from presented work is that by using the described model with gamma measurements taken from an UAV it is possible to localize a radioactive source within a few minutes. Field tests showed, that single gamma radiation sources could be localized very precisely even in scenarios simulating a radiation source located inside a collapsed building. Moreover, a 3D terrain map of the observed area can be presented in real-time. This can greatly improve the efficiency of localizing radioactive sources in destructed areas that are difficult to access and minimize health risks and the risks for civilians. The presented model supports only single sources. For multiple sources the model will still produce results, but the source location will be confined to a larger area and likely be in the range between the two sources. However, if these sources are close together the location map would provide a good hint of the true positions.

In future work the model should be extended to detect multiple sources as well to increase the practical use of such a system.

\section{ACKNOWLEDGEMENTS (OPTIONAL)}

SecuRescue has been funded by the Austrian security research programme KIRAS of the Federal Ministry for Transport, Innovation and Technology (bmvit).

\section{REFERENCES}

Ardiny H, Witwicki S, Mondada F., 2019: Autonomous Exploration for Radioactive Hotspots Localization Taking Account of Sensor Limitations. Sensors (Basel), 19(2), 292. doi:10.3390/s19020292.

Branko Ristic, Mark Morelande, Ajith Gunatilaka, 2010: Information driven search for point sources of gamma radiation. Signal Process. 90(4), 1225-1239.

Gabrlik P., Lazna T., 2018: Simulation of Gamma Radiation Mapping Using an Unmanned Aerial System. ScienceDirect 51(6), 256-262. doi.org/10.1016/j.ifacol.2018.07.163

Gordon C., Shoemaker A., Kochersberger K., Tokekar P., McLean L., Leonessa A., 2017: Radiation search operations using scene understanding with autonomous UAV and UGV. Journal of Field Robotics 34(8), 1450-1468.

Meng, Xuelian and Currit, Nate and Zhao, Kaiguang, 2010: Ground Filtering Algorithms for Airborne LiDAR Data: A Review of Critical Issues. Remote Sensing, 2(3), 833-860. 